\title{
TOTALLY NONCONNECTED IM KLEINEN CONTINUA
}

\author{
EDWARD E. GRACE
}

If a connected topological space $T^{\prime}$ is not connected im kleinen at the point $p$ of $T^{\prime}$, then there is some open set $D^{\prime}$ containing $p$ such that $p$ is a boundary point of the $p$-component of $D^{\prime}$.

This paper shows that much stronger conditions than those above hold at some points of a Baire topological continuum ${ }^{1}$ which is not connected im kleinen at any point of a certain domain intersection subset. Limits to results of this kind are shown by an example of a bounded plane continuum that is connected im kleinen at each point of a dense inner limiting (i.e., $G_{\delta}$ ) subset but is not locally connected at any point.

Definition. A topological continuum $T^{\prime}$ is totally nonconnected im kleinen on a subset $A$ of $T^{\prime}$ if $T^{\prime}$ is not connected im kleinen at any point of $A$ (i.e., if each point $p$ of $A$ is contained in some open subset $U$ of $T^{\prime}$ such that $p$ is a boundary point, relative to $T^{\prime}$, of the $p$ component of $U$ ).

Definition. If (1) $T^{\prime}$ is a topological continuum, (2) $Z^{\prime}$ is the least cardinal number of any topological basis for $T^{\prime}$, (3) the set $U$ is an open subset of $T^{\prime}$, and (4) the subset $A$ of $U$ is the nonvacuous common part of not more than $Z^{\prime}$ open subsets of $T^{\prime}$, each dense in $U$, then $A$ is a dense-domain intersection subset of $U$ (relative to $\left.T^{\prime}\right)$. If, in addition to (1), (2) and (3), $U$ is not the sum of $Z^{\prime}$, or fewer than $Z^{\prime}$, closed nowhere dense subsets of $T^{\prime}$, then $T^{\prime}$ is Baire topological on $U$. If $T^{\prime}$ is Baire topological on each open subset of $U$, then $T^{\prime}$ is locally Baire topological on $U$.

THEOREM 1. If the topological continuum $T^{\prime}$ is Baire topological on the open subset $U$, then $U$ contains an open subset $V$ such that $T^{\prime}$ is locally Baire topological on $V$.

Proof. Let $R_{1}^{\prime}, R_{2}^{\prime}, \cdots, R_{i}^{\prime}, \cdots$ be a most economical wellordering of a basis for $T^{\prime}$ of minimum cardinality. Assume $T^{\prime}$ is not locally Baire topological on any open subset of $U$. Then there is a subsequence $R_{N(1)}^{\prime}, R_{N(2)}^{\prime}, \cdots, R_{N(i)}^{\prime}, \cdots$ of $R_{1}^{\prime}, R_{2}^{\prime}, \cdots, R_{i}^{\prime}, \cdots$ such that, for each $i$, there is a dense-domain intersection subset $I_{i}$ of $T^{\prime}$ such that $I_{i} \cdot R_{N(i)}^{\prime}=0$ and each open subset of $U$ contains some term of $R_{N(1)}^{\prime}, R_{N(2)}^{\prime}, \cdots, R_{N(i)}^{\prime}, \cdots$. But $\prod_{i} I_{i}$ is the common part

Presented to the Society January 28, 1958; received by the Editors December 16, 1957.

1 The terminology of this paper is that of [1]. 
of not more than $Z^{\prime}$ dense-domain intersection subsets of $T$, where $Z^{\prime}$ is the cardinality of $R_{1}^{\prime}, R_{2}^{\prime}, \cdots, R_{i}^{\prime}, \ldots$. Consequently $[1$, Theorem 3$]$, the set $\prod_{i} I_{i}$ is a dense-domain intersection subset of $T$. But [1, Theorem 1], the closure of any dense-domain intersection subset of $T$ contains an open subset of $U$, and therefore, the closure of $\prod_{i} I_{i}$ contains an open subset of $U$. This is a contradiction.

Standing notation. $T$ is a topological continuum which is locally Baire topological on the open set $D$ and is totally nonconnected im kleinen on a dense-domain intersection subset $I$ of $D$. The least cardinal number of a basis for $T$ is $Z$. The collection $R$ of regions is a basis for $T$ of cardinality $Z$. The sequence $R_{1}, R_{2}, \cdots, R_{i}, \cdots$, is a most economical well-ordering of $R$.

Lemma 1. Let $U$ be an open subset of $D$ and let $S$ be the set of all points $p$ such that $p$ is in $\bar{U}-U$ or $p$ is in $U$ and $p$ is a boundary point of the p-component of $U$. Then $S$ is closed.

Proof. Assume there is a point $q$ in $\bar{S}$ which is not in $S$. Then $q$ is in $U$ since $\bar{U} \supset \bar{S}$ and each point of $\bar{U}-U$ is in $S$. Therefore, each region containing $q$ contains a point $r$ of $S \cdot U$ and hence contains a point of $U$ not in the $q$-component of $U$. Consequently, $q$ is in $S$. This is a contradiction and so $S$ is closed.

Lemma 2. Let $V$ be an open subset of $D$. Then $V$ contains an open set no component of which contains an open set.

Proof. Let $R_{M(1)}, R_{M(2)}, \cdots, R_{M(i)}, \cdots$ be the subsequence of $R_{1}, R_{2}, \cdots, R_{i}, \cdots$ consisting of those terms contained in $V$. For each $i$, let $S_{i}$ be the set related to $R_{M(i)}$ as $S$ is related to $U$ in Lemma 1. The continuum $T$ is totally nonconnected im kleinen on the densedomain intersection subset $I \cdot V$ of $V$ and therefore $\sum_{i} S_{i} \supset I \cdot V$. But, since $I$ is a dense-domain intersection subset of $D$, the set $D-I$ is contained in the sum of not more than $Z$ closed sets, each nowhere dense in $D$. Hence, if each term of $S_{1}, S_{2}, \ldots$ were nowhere dense, then $V$ would be contained in the sum of not more than $Z$ closed, nowhere dense subsets of $T$. But that can not be, since $T$ is Baire topological on $V$. Consequently, for some $i$, the set $S_{i}$ contains an open set $V^{\prime}$. By definition of $S_{i}$, no component of the open set $V \cdot V^{\prime}$ can contain an open set.

TheOREM 2. The open set $D$ contains an open set $D^{\prime}$, dense in $D$, no component of which contains an open set.

Proof. Let $D_{1}$ be an open subset of $D$, no component of which contains an open set. If $i$ is an ordinal greater than 1 such that the 
closure of $\sum_{j<i} D_{j}$ does not contain $D$, then let $D_{i}$ be an open subset of the complement, in $D$, of the closure of $\sum_{j<i} D_{j}$, no component of which contains an open set. Let $D^{\prime}=\sum_{j} D_{j}$. Then $D^{\prime}$ is an open subset of $D$, dense in $D$, no component of which contains an open set.

Theorem 3. Let the topological continuum $T^{\prime}$ be locally Baire topological on the open set $V$. Then $T^{\prime}$ is totally nonconnected im kleinen on a dense-domain intersection subset of $V$ if and only if there is an open subset of $V$, dense in $V$, no component of which contains an open set.

Theorem 4. If $T$ is regular then $T$ contains a dense-domain intersection subset $J$ of $D$, contained in $I$, such that if $p$ is a point of $J$ and $T$ is nonaposyndetic at $p$ with respect to $T-\bar{R}_{i}$, where $R_{i}$ contains $p$, then the p-component of $\bar{R}_{i}$ does not contain an open set.

Proof. The collection $G$ of complements of closures of regions of $R$ has the $Z$-domain property and $T$ is totally nonaposyndetic on $I$ with respect to $G$. Let $P$ be a dense subset of $T$ of cardinality not greater than $Z$. The author has proved [1, Theorem 8] that under these circumstances there is a dense-domain intersection subset $J$ of $D$, contained in $I$, such that if $q$ is a point in $J$ and $T$ is nonaposyndetic at $q$ with respect to $T-\bar{R}_{i}$ of $G$ then $T-\bar{R}_{i}$ cuts (weakly) $q$ from each point of $P \cdot \bar{R}_{i}$. In which case, the p-component of $\bar{R}_{i}$ does not contain an open set.

The following example of a plane continuum, each connected open subset of which is dense in it, shows that Theorem 3 does not hold if "totally nonconnected im kleinen on a dense-domain intersection subset of $V$ " is replaced by "not locally connected at any point of $V$ "; even in the case where $V=T^{\prime}$.

Let $H_{1}, H_{2}, \cdots$ be the sequence of closed plane point sets defined by induction as follows. Let $S$ denote the unit disk and let $H_{1}$ be the logical sum of the closed (topological) disk sequence $J_{1}, J_{2}, \cdots$ indicated in Figure 1. The sum of the straight portions of $J_{1}, \cdots, J_{4}$ is the limiting set of $J_{1}, J_{2}, \cdots$.

Assume $H_{i}$ to be defined and let $J_{1}^{\prime}, J_{2}^{\prime}, \cdots$ be a counting of the closed disks maximal in $H_{i}$. For each natural number $j$, there exist two points $r$ and $s$ of $J_{j}^{\prime}$ such that $r+s$ cuts $J_{j}^{\prime}$ from $H_{i}-J_{j}^{\prime}$ in $H_{i}$ and such that if a single point $t$ does the same cutting then $t=r$. Let $f_{j}$ be a homeomorphism of $S$ onto $J_{j}^{\prime}$ such that $f_{j}(p)=r$ and $f_{j}(q)=s$. Let $H_{i+1}=\sum_{j} f_{j}\left(H_{1}\right)$. The homeomorphisms of $S$ onto the closed maximal disks of the sets $H_{2}, H_{3}, \cdots$ are chosen in such a way that the diameters of disks in $H_{1}, H_{2}, \cdots$ are eventually as small as one wishes. 


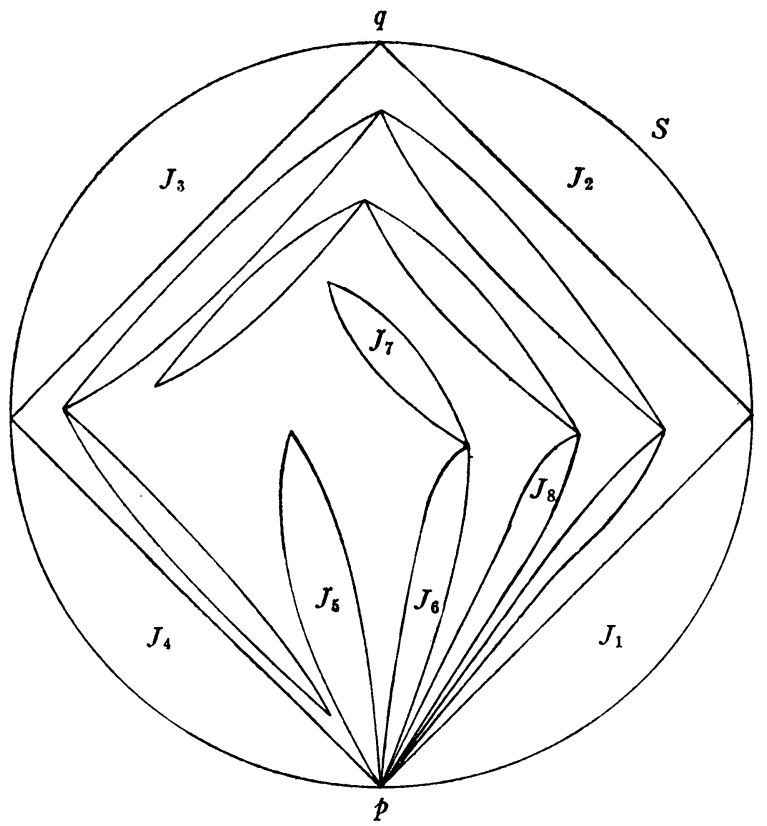

FIG. 1

Let $H=\prod_{i} H_{i}$. It is clear that $H$ is not locally connected at any point but $H$ is connected im kleinen at each point of the dense inner limiting subset of $H$ consisting of the points that, for each $i$, are interior points (in the plane) of $H_{i}$. Hence $H$ does not contain any open set no component of which contains an open set.

\section{BIBLIOGRAPHY}

1. E. E. Grace, The existence of cut sets in totally nonaposyndetic continua, Proc. Amer. Math. Soc. vol. 9 (1958) pp. 98-104.

2. J. L. Kelley, General topology, New York, 1955.

3. R. L. Moore, Foundations of point set theory, Amer. Math. Soc. Colloquium Publications, vol. 13, 1932.

EMORY UNIVERSITY 